UUITP-06/04

hep-th/0402192

\title{
Type 0A 2D Black Hole Thermodynamics and the Deformed Matrix Model
}

\author{
Ulf H. Danielsson ${ }^{1}$, James P. Gregory ${ }^{2}$, Martin E. Olsson ${ }^{3}$, \\ Peter Rajan ${ }^{4}$ and Marcel Vonk ${ }^{5}$ \\ Institutionen för Teoretisk Fysik, Box 803, SE-751 08 Uppsala, Sweden \\ ${ }^{1}$ ulf.danielsson@teorfys.uu.se \\ 2 james.gregory@teorfys.uu.se \\ ${ }^{3}$ martin.olsson@teorfys.uu.se \\ ${ }^{4}$ peter.rajan@teorfys.uu.se \\ ${ }^{5}$ marcel.vonk@teorfys.uu.se
}

\begin{abstract}
Recently, it has been proposed that the deformed matrix model describes a twodimensional type $0 \mathrm{~A}$ extremal black hole. In this paper, the thermodynamics of $0 \mathrm{~A}$ charged non-extremal black holes is investigated. We observe that the free energy of the deformed matrix model to leading order in $1 / q$ can be seen to agree to that of the extremal black hole. We also speculate on how the deformed matrix model is able to describe the thermodynamics of non-extremal black holes.
\end{abstract}

February 2004 


\section{Introduction}

Recently, the work in [1, 2, 3] has revived the interest in matrix model descriptions of noncritical string theories in two dimensions. In particular, it has been argued in [2, 3. that the two-dimensional type $0 \mathrm{~A}$ and type $0 \mathrm{~B}$ string theories each are dual to matrix models which are nonperturbatively stable.

The so-called deformed matrix model which was suggested to describe the twodimensional type 0A string theory had appeared in the literature long before these developments - in 4 it was proposed that this matrix model describes two-dimensional string theory in a black hole background. For further developments of this see [5-10]. Very recently [11, 12, it was argued that both of these identifications are essentially correct, and that type 0A string theory in the presence of a background flux $q$ can be viewed as describing an extremal two-dimensional black hole of charge $q$.

In this paper, we give further evidence for this claim by calculating the thermodynamical properties of non-extremal two-dimensional charged black holes. In particular, we calculate the free energy, entropy and temperature of these black holes. We show that in the extremal limit, this free energy exactly matches the matrix model free energy. This supports the observation made in [12] where it was found that the ADM mass of the extremal black hole equals the free energy of the 0A matrix model.

The next natural question to ask is whether there is also a matrix model description of non-extremal two-dimensional black holes. In this respect, the cutoff dependence we find on the space-time side is quite suggestive. Motivated by this, we discuss a possible modification of the deformed matrix model based on the work in 13$]$.

This paper is organized as follows. In section 2, we discuss the thermodynamics of the two-dimensional charged black hole and calculate the free energy by evaluating the Euclidean action. In section $[3$, we begin by calculating the free energy of the type 0A matrix model, finding a result which exactly matches the result of the previous section in the extremal case. Subsequently, we discuss in more detail the relation between the two sides of the duality. In particular, we describe how the deformed matrix model could be modified in order to take the temperature dependence of the non-extremal black hole into account. We conclude in section 4 .

Note added. In preparing this paper for submission, we received the paper [14, where the thermodynamic properties of the two-dimensional charged black hole are also calculated. While our results on the thermodynamics on the space-time side agree with those in [14, the conclusions on the comparision to the matrix model seem to differ. 


\section{Free Energy and Thermodynamics of the Black Hole}

\subsection{The Black Hole Solution}

The starting point for our analysis will be the space-time effective action for type 0A string theory [15, 16, 11, 12] in the presence of a RR flux $q$ and zero tachyon field, ${ }^{1}$

$$
S=-\int \mathrm{d}^{2} x \sqrt{-g}\left[e^{-2 \Phi}\left(\mathcal{R}+4(\nabla \Phi)^{2}+4 k^{2}\right)+\Lambda\right]
$$

where $\Phi$ is the dilaton field and the constants $k$ and $\Lambda$ are given by ${ }^{2}$

$$
k=\sqrt{\frac{2}{\alpha^{\prime}}} \quad \Lambda=\frac{-q^{2}}{4 \pi \alpha^{\prime}} .
$$

The equations of motion following from this action are

$$
\begin{aligned}
& \mathcal{R}_{\mu \nu}-\frac{\Lambda}{2} e^{2 \Phi} g_{\mu \nu}+2 \nabla_{\mu} \nabla_{\nu} \Phi=0 \\
& \mathcal{R}+4 k^{2}+4 \nabla^{2} \Phi-4(\nabla \Phi)^{2}=0
\end{aligned}
$$

Denoting the space-time coordinates by $(t, \phi)$, one finds the following one-parameter family of solutions [16, 11, 12,

$$
\begin{aligned}
d s^{2} & =-l(\phi) \mathrm{d} t^{2}+\frac{1}{l(\phi)} \mathrm{d} \phi^{2} \\
\Phi & =-k \phi
\end{aligned}
$$

where

$$
l(\phi)=1-e^{-2 k \phi}\left(\frac{m}{k}-\frac{\Lambda}{2 k} \phi\right) .
$$

The nature of such solutions depends on the value of the parameter $m$ in relation to its critical value

$$
m_{\mathrm{ext}}=\frac{\Lambda}{4 k} \ln \left(\frac{-\Lambda}{4 k^{2} e}\right) .
$$

For $m<m_{\text {ext }}$ the space-time has a naked singularity and these solutions will not be of interest to us. For $m \geq m_{\text {ext }}$ the solution represents a charged black hole whose horizon, $\phi_{H}$, is given by the largest zero of $l(\phi)$. Whilst $l(\phi)$ generically has two zeros for the black hole space-time, these coincide when the lower bound on $m$ is saturated and at this point the black hole is extremal.

\footnotetext{
${ }^{1}$ The sign of the action varies in the literature but this choice of sign depends on the definition of the spatial coordinate in the measure.

${ }^{2}$ We note that, in comparison to the actions given in 12, 14, our value of $\Lambda$ differs by a factor of 2 . This is because we choose only to include contributions to the $\Lambda$ term from either the electric or magnetic sector of D0 branes, which allows for a generalization to a non-zero tachyon field. For a discussion of this point we refer the reader to, for example, 3].
} 


\subsection{Basic Black Hole Thermodynamics}

Our two-dimensional black holes are parametrized by the mass parameter $m$. However, it often becomes easier to parametrize by the horizon radius $\phi_{H}$ instead. Using $l\left(\phi_{H}\right)=0$, these two quantities are related by the one-to-one correspondence

$$
m=k e^{2 k \phi_{H}}+\frac{\Lambda}{2} \phi_{H} .
$$

The temperature of the black hole can be defined by moving to the Euclidean section $t \rightarrow i \tau$. In so doing the horizon develops a bolt singularity which must subsequently be removed by compactifying the Euclidean time direction $\tau$ according to [17]

$$
\tau \sim \tau+\beta \quad \text { where } \quad \beta=\frac{4 \pi}{l^{\prime}(\phi)} .
$$

The temperature of the black hole is then defined by

$$
T=\frac{1}{\beta}=\frac{1}{8 \pi k}\left(4 k^{2}+\Lambda e^{-2 k \phi_{H}}\right)
$$

The relation now of course allows us to parametrize the black hole space-time in terms of $T$ instead by using the one-to-one mapping

$$
\phi_{H}=-\frac{1}{2 k} \ln \left(\frac{8 \pi k T-4 k^{2}}{\Lambda}\right) .
$$

In what follows we will refer to horizon radius of the extremal black hole $\phi_{H}(T=0)$ as $\phi_{\text {ext }}$.

The mass of the black hole space-time can be evaluated via the ADM formalism (see for example [18]). This requires a reference space-time for which we take the extremal black hole. The ADM energy is then given by

$$
\begin{aligned}
E_{\mathrm{ADM}}=M-M_{\mathrm{ext}} & =\left[-4 k e^{2 k \phi}\left(l(\phi)-\sqrt{l(\phi) l_{\mathrm{ext}}(\phi)}\right)\right]_{\phi \rightarrow \infty} \\
& =\left(2 m-\Lambda \phi_{c}\right)-\left(2 m_{\mathrm{ext}}-\Lambda \phi_{c}\right),
\end{aligned}
$$

where $M=2 m-\Lambda \phi_{c}$ is the mass of the black hole space-time including a divergent volume term. We have regulated this contribution with an infrared cutoff $\phi_{c}$ in the spatial direction $\phi$ and ignored terms which vanish in the large $\phi_{c}$ limit.

To completely determine the thermodynamics of the black hole it now remains to calculate the free energy from which one can also derive the entropy.

\subsection{Free Energy from the Euclidean Action}

Our objective now is to calculate the free energy by using Euclidean Quantum Gravity techniques. Of course, one may be tempted to calculate the entropy and free energy directly by integrating up the second law of thermodynamics, but the integration 
constants which arise could be of importance so we will avoid this problem in what follows.

The free energy, $F$, can be defined in terms of the on-shell value of the Euclidean action, $I$, through the simple relation

$$
F=\frac{I}{\beta}
$$

We thus proceed to evaluate the Euclidean action and the remaining thermodynamic properties of our solution will be revealed.

In moving to the Euclidean section, $t \rightarrow i \tau$, one must supplement an action with a Gibbons-Hawking boundary term which allows the use of a well-defined variational principle [17]. In doing so our complete Euclidean action now becomes

$$
I=-\int_{\mathcal{M}} \sqrt{g}\left[e^{-2 \Phi}\left(\mathcal{R}+4(\nabla \Phi)^{2}+4 k^{2}\right)+\Lambda\right]-2 \int_{\partial \mathcal{M}} \sqrt{h} e^{-2 \Phi} K,
$$

where $h_{a b}$ is the induced metric on the boundary and $K$ is the trace of the second fundamental form.

Formally evaluating the action for our solution will give infinity as we intend to evaluate over all Euclidean space, i.e. the entire region outside of the black hole horizon. To deal with this a background subtraction is required. In choosing a reference background it must be one which belongs to the ensemble of black hole solutions that are under consideration, i.e. in this case it must also have charge $q$. Therefore the natural background to consider is the extremal black hole defined by $m=m_{\text {ext }}$. Our regularized action then becomes

$$
\begin{aligned}
I=- & \left(\int_{\mathcal{M}}-\int_{\mathcal{M}_{\mathrm{ext}}}\right) \sqrt{g}\left[e^{-2 \Phi}\left(\mathcal{R}+4(\nabla \Phi)^{2}+4 k^{2}\right)+\Lambda\right] \\
& -2\left(\int_{\partial \mathcal{M}}-\int_{\partial \mathcal{M}_{\mathrm{ext}}}\right) \sqrt{h} e^{-2 \Phi} K .
\end{aligned}
$$

In order to match the black hole with the extremal black hole reference background at spatial infinity the limits of integration must be chosen with care. Our intention is to evaluate over all space-time, but this is achieved by formally integrating up to some cutoff $R$ in the $\phi$ coordinates for both space-times and then letting $R \rightarrow \infty$. The $\tau$ integration then proceeds as follows. Whilst for the black hole we integrate $\tau$ from 0 to $\beta$, the geometry of the reference background must match the black hole geometry at the cutoff surface $\phi=R$. This requires us to choose a period of integration for the $\tau$ coordinate of the extremal black hole given by the following relation ${ }^{3}$

$$
\beta \sqrt{g_{\tau \tau}^{B H}(R)}=\beta_{\mathrm{ext}} \sqrt{g_{\tau \tau}^{\mathrm{ext}}(R)}
$$

\footnotetext{
${ }^{3}$ This is valid since for the extremal black hole there is no bolt singularity at the horizon and so one may formally assign any desired period to the Euclidean time [19, 20].
} 
Evaluating $\beta_{\text {ext }}$ at large $R$ we find

$$
\frac{\beta_{\mathrm{ext}}}{\beta}=1-\frac{m-m_{\mathrm{ext}}}{2 k} e^{-2 k R}+\mathcal{O}\left(e^{-4 k R}\right) .
$$

Given that the limits of integration are now established we may turn to the integrands. Evaluating the bulk integrand we find

$$
\mathcal{L}_{\text {bulk }}=3 \Lambda+8 k^{2} e^{2 k \phi} .
$$

The boundary integrand needs a little bit of technology. We pick a unit normal to the boundary $n^{\mu}=\left(0,1 / \sqrt{g_{\phi \phi}}\right)$. Then $K_{a b}$ is the Lie derivative of the induced metric with respect to this normal, i.e.

$$
K_{a b}=h_{(a}^{c} h_{b)}^{d} \partial_{c} n_{d}
$$

In our case the only nonzero component of $K$ is the $\tau \tau$-component and the trace of $K$ can be subsequently found,

$$
K_{\tau \tau}=\frac{1}{2} \sqrt{l(\phi)} l^{\prime}(\phi) \Rightarrow \sqrt{h} K=\frac{l^{\prime}(\phi)}{2} .
$$

We can now evaluate the integrand of the boundary integral to obtain

$$
\mathcal{L}_{\text {bdry }}=\bar{m}-\frac{R \Lambda}{2}+\frac{\Lambda}{2 k},
$$

where $\bar{m}$ is $m$ or $m_{\text {ext }}$ respectively.

We now have all the pieces we need to evaluate the Euclidean action of our solution

$$
\begin{aligned}
I=- & \left(\int_{0}^{\beta} \mathrm{d} \tau \int_{\phi_{H}}^{R} \mathrm{~d} \phi-\int_{0}^{\beta_{\text {ext }}} \mathrm{d} \tau \int_{\phi_{\text {ext }}}^{R} \mathrm{~d} \phi\right) \mathcal{L}_{\text {bulk }}-2\left(\int_{0}^{\beta} \mathrm{d} \tau-\int_{0}^{\beta_{\text {ext }}} \mathrm{d} \tau\right) \mathcal{L}_{\text {bdry }} \\
=-\beta & {\left[\left(3 \Lambda R+4 k e^{2 k R}\right)\left(1-\frac{\beta_{\text {ext }}}{\beta}\right)-\left(3 \Lambda \phi_{H}+4 k e^{2 k \phi_{H}}\right)+\frac{\beta_{\text {ext }}}{\beta}\left(3 \Lambda \phi_{\text {ext }}+4 k e^{2 k \phi_{\text {ext }}}\right)\right] } \\
& -2 \beta\left[m-\frac{R \Lambda}{2}+\frac{\Lambda}{2 k}-\frac{\beta_{\text {ext }}}{\beta}\left(m_{\text {ext }}-\frac{R \Lambda}{2}+\frac{\Lambda}{2 k}\right)\right] .
\end{aligned}
$$

Taking $R \rightarrow \infty$ we obtain the action of our black hole space-time relative to the extremal black hole reference background

$$
I=\beta \Lambda\left(\phi_{H}-\phi_{\mathrm{ext}}\right) .
$$

The free energy of the solution now follows. Given that we are considering an ensemble of fixed charge black hole solutions, what we have actually calculated is the Helmholtz free energy for the canonical ensemble. We therefore know that this is given by the following relation

$$
F=\frac{I}{\beta}=E-T S
$$

where $E=M-M_{\text {ext }}$. 


\subsection{Black Hole Entropy}

In order to calculate the entropy in the simplest manner the free energy should first be expressed in terms of temperature alone

$$
F=-\frac{\Lambda}{2 k} \ln \left(1-\frac{2 \pi}{k} T\right) .
$$

Notice that the free energy for the extremal black hole is zero as we would expect since we have the extremal black hole as a reference background.

The black hole entropy then follows from the standard thermodynamic relation relating Helmholtz free energy to entropy

$$
\begin{aligned}
S=-\frac{\partial F}{\partial T} & =-\frac{\pi \Lambda}{k^{2}}\left(1-\frac{2 \pi}{k} T\right)^{-1} \\
& =4 \pi e^{2 k \phi_{H}}
\end{aligned}
$$

It is important to observe that the entropy of the extremal black hole is non-zero. Furthermore, we comment that this entropy can indeed be obtained by integrating up the second law of thermodynamics given our value for the temperature and the ADM mass of the black hole. Expressing both as functions of $\phi_{H}$ and integrating over what seems the most natural range for this radial coordinate, $\left[\phi_{H}, \infty\right]$, the correct constant of integration is obtained.

As an aside, note that we can express the entropy (2.27) in a familiar form,

$$
S=4 \pi e^{2 k \phi_{H}}=\frac{4 \pi}{e^{2 \Phi_{H}}}=\frac{A}{4 G_{N}}
$$

where Newton's constant, $G_{N}$, is expressed in terms of the dilaton at the horizon, $G_{N}=e^{2 \Phi_{H}} / 16 \pi$. Here we are using the natural value $A=1$ for the area of the black hole horizon as seen from one side of the singularity.

\subsection{Non-zero Free Energy of the Extremal Black Hole}

Given the fact that the free energy we have calculated is the Helmholtz free energy, we have been able to express this in terms of the basic thermodynamic quantities of the black hole space-time (2.25):

$$
F=M-M_{\mathrm{ext}}-T S
$$

Obviously such a free energy includes a contribution from the reference space-time of our subtraction scheme, but this subtraction may be easily removed. We can calculate the free energy of the black hole space-time alone by merely adding back on the contribution from the mass of the extremal black hole

$$
F_{\mathrm{BH}}=F+M_{\mathrm{ext}} \equiv M-T S
$$


The resulting free energy is given by

$$
\begin{aligned}
F_{\mathrm{BH}}=\Lambda \phi_{H}-\Lambda \phi_{\mathrm{ext}}+2 m_{\mathrm{ext}}-\Lambda \phi_{c} & =\Lambda\left(\phi_{H}-\phi_{c}\right)-\frac{\Lambda}{2 k} \\
& =-\frac{\Lambda}{2 k} \ln \left(e^{1+2\left(\Phi_{H}-\Phi_{c}\right)}\right),
\end{aligned}
$$

where in the last line we have used $\Phi=-k \phi$. This free energy can also be expressed in terms of the temperature as

$$
F_{\mathrm{BH}}=-\frac{\Lambda}{2 k} \ln \left(\frac{-4 k^{2} e}{\Lambda}\left[1-\frac{2 \pi}{k} T\right] e^{-2 \Phi_{c}}\right) .
$$

This is obviously the free energy presented earlier up to the additive constant of the extremal black hole mass so the entropy is unaffected.

Most importantly to us now, we have a non-zero free energy for the case of the extremal black hole which we shall demonstrate is reproduced in the matrix model,

$$
F_{\mathrm{BH}}(T=0)=-\frac{\Lambda}{2 k} \ln \left(\frac{-4 k^{2} e}{\Lambda} e^{-2 \Phi_{c}}\right)=-\frac{q^{2}}{16 \pi} \sqrt{\frac{2}{\alpha^{\prime}}} \ln \left(\frac{q^{2}}{32 \pi e} e^{2 \Phi_{c}}\right),
$$

where the definitions (2.2) have been inserted.

\section{The Matrix Model Free Energy}

Let us now turn to the deformed matrix model and calculate the free energy there. ${ }^{4}$ We will find that, to leading order in $1 / q$, the extremal value of the black hole free energy (2.33) precisely matches the matrix model free energy calculated below.

We shall also discuss how the matrix model can be modified in order to reproduce the $T$-dependent free energy representing the non-extremal black hole.

\subsection{Free Energy from the Matrix Model}

It is well known that the free energy of the matrix model can be written as

$$
F_{\mathrm{MM}}=\int_{-\varepsilon_{c}}^{-\mu} \varepsilon \rho(\varepsilon) d \varepsilon
$$

where the density of the number of states is given by

$$
\rho(\varepsilon)=-\frac{1}{\pi} \operatorname{Im} \sum_{n=0} \frac{1}{E_{n}+\varepsilon}
$$

at zero temperature. $-\mu$ is the position of the Fermi sea, and $-\varepsilon_{c}$ is a cutoff.

\footnotetext{
${ }^{4}$ Such calculations have previously been done in, e.g., $[6]$.
} 
Following [5] we may formally continue $\alpha^{\prime} \rightarrow-\alpha^{\prime}$ in order to have a right side up harmonic oscillator. We then have the following Schrödinger equation to solve:

$$
\left(-\frac{1}{2} \frac{d^{2}}{d x^{2}}+\frac{1}{4 \alpha^{\prime}} x^{2}+\frac{\eta}{2 x^{2}}\right) \psi(x)=E \psi(x),
$$

where $\eta=q^{2}-1 / 4$. Using this, the energy eigenvalues of the original problem can be shown to be

$$
E_{n}=\frac{i}{2 \sqrt{2 \alpha^{\prime}}}\left(2 n+1+2 a_{n}\right)
$$

where

$$
a_{n}=-(-1)^{n}\left(-\frac{1}{2}+\sqrt{\frac{1}{4}+\eta}\right) .
$$

For $q \geq 0$, we must throw away the even parity solutions leaving only the odd levels given by

$$
E_{2 l+1}=\frac{i}{2 \sqrt{2 \alpha^{\prime}}}(4 l+3-1+2 q)=\frac{i}{\sqrt{2 \alpha^{\prime}}}(2 l+1+q) .
$$

To leading order in large $q$ we find

$$
\rho(\varepsilon)=-\frac{1}{\pi} \operatorname{Im} \sum_{l=0} \frac{1}{\frac{i}{\sqrt{2 \alpha^{\prime}}}(2 l+1+q)+\varepsilon} \sim-\frac{1}{2 \pi} \sqrt{\frac{\alpha^{\prime}}{2}} \ln \left(\frac{q^{2}}{4}+\frac{\alpha^{\prime}}{2} \varepsilon^{2}\right)+\text { const. }
$$

where the constant depends on the cutoff $\varepsilon_{c}$ but is independent of $q$. Using this we find a free energy (at $\mu=0$ ) given by

$$
F_{\mathrm{MM}}=-\frac{q^{2}}{16 \pi} \sqrt{\frac{2}{\alpha^{\prime}}} \ln \left(\frac{q^{2}}{4 e} \frac{2}{\alpha^{\prime} \varepsilon_{c}^{2}}\right)+\ldots,
$$

up to terms of higher order in $1 / \varepsilon_{c}$ or independent of $q$.

\subsection{Comparing the Deformed Matrix Model with the Black Hole}

If we compare (3.8) with the free energy in equation (2.33), derived from the spacetime point of view, we see that the numerical coefficient in front of the logarithm is in perfect agreement. That is, the cutoff independent non-analytical (in $q$ ) parts of the free energies are identical. The agreement carries over also to the analytic cutoff dependent part of the expression provided that we relate the spatial spacetime cutoff $\phi_{c}$ to the energy cutoff $\varepsilon_{c}$ in the matrix model. The latter can also be understood as an IR-cutoff in the matrix eigenvalue coordinate $x$. Even though the cutoffs are related in a natural way, it is well known that the relation between the matrix eigenvalue coordinate and space-time is quite complicated and given by a nonlocal integral transform. Nevertheless, it is reassuring to see the correspondence. 
While it is gratifying that we obtain this agreement on the level of the free energy at zero temperature, it would be nice to be able to reproduce the full temperature dependent free energy using the deformed matrix model. In this context one should note that the temperature dependence of (2.31) is of an extremely simple form. Indeed, all of the temperature dependence comes from the way in which the spatial volume depends on the position of the horizon. ${ }^{5}$ This can, possibly, be a clue towards a complete understanding of the correspondence from the point of view of the matrix model.

Unfortunately, it is not enough to turn on a temperature in the deformed matrix model simply by compactifying Euclidean time. The resulting free energy will not be of the form (2.31) and will not give rise to the correct entropy. The most natural interpretation of this observation is that the naive way of turning on a temperature just corresponds to placing the extremal black hole in an external heat bath without changing the mass.

Instead it is reasonable to expect that we need yet another parameter to deform the matrix model. An interesting candidate is found if we make use of the non-singlet states of the matrix model as is done in [13. It is claimed in that paper that the fugacity of the vortices is a measure of the mass of a Witten type uncharged black hole 21. In our case we expect the fugacity to be a measure of the departure from extremality, and, as a consequence, the calculations of [13] would have to be redone for the deformed matrix model. In principle we would then have two free parameters: the fugacity and the temperature. In order to reproduce the correct thermodynamics, however, there needs to be an extra relation analogous to the space-time relation between the temperature and the mass. It would be interesting to see how this comes about in the matrix model. ${ }^{6}$

\section{Conclusions}

In this paper we have discussed the thermodynamics of the two-dimensional type 0A black hole. In particular, using the Euclidean approach, we calculated the (nonextremal) classical free energy (2.31), from which all the other quantities of interest follow in the usual way.

In comparing the space-time results to the conjectured dual $0 \mathrm{~A}$ matrix model we found that the free energies agree in the extremal limit. It would quite naturally be very interesting to see if the agreement between the free energies still holds away from extremality. In particular one would like to be able to calculate the black hole entropy directly from the matrix model. The correct matrix model description of this non-extremal black hole is still an open question.

However, we believe that the evidence presented here (see also [11, 12]) in favour of the duality between the deformed matrix model and the 0A black hole is an important

\footnotetext{
${ }^{5}$ One is tempted to write the cutoff on the matrix model side as $x_{H}^{4} / x_{c}^{4}$ in a similar fashion.

${ }^{6}$ One should note that in case of the uncharged black hole the temperature is independent of the mass. Compare (2.11) which for $q=0$ gives $T=\frac{k}{2 \pi}$.
} 
step towards a better understanding of this issue.

\section{Acknowledgments}

We would like to thank A. Padilla for correspondence. UD is a Royal Swedish Academy of Sciences Research Fellow supported by a grant from the Knut and Alice Wallenberg Foundation. The work was also supported by the Swedish Research Council (VR).

\section{References}

[1] J. McGreevy and H. Verlinde, Strings from tachyons: the $c=1$ matrix reloaded, JHEP 0312 (2003) 054 hep-th/0304224.

[2] T. Takayanagi and N. Toumbas, A matrix model dual of type OB string theory in two dimensions, JHEP 0307 (2003) 064 hep-th/0307083.

[3] M. R. Douglas, I. R. Klebanov, D. Kutasov, J. Maldacena, E. Martinec and N. Seiberg, A new hat for the $c=1$ matrix model hep-th/0307195.

[4] A. Jevicki and T. Yoneya, A deformed matrix model and the black hole background in two-dimensional string theory, Nucl. Phys. B411 (1994) 64-96 hep-th/9305109.

[5] U. H. Danielsson, A matrix model black hole, Nucl. Phys. B410 (1993) 395-406 hep-th/9306063.

[6] K. Demeterfi and J. P. Rodrigues, States and quantum effects in the collective field theory of a deformed matrix model, Nucl. Phys. B415 (1994) 3-28 hep-th/9306141.

[7] K. Demeterfi, I. R. Klebanov and J. P. Rodrigues, The exact $S$ matrix of the deformed $c=1$ matrix model, Phys. Rev. Lett. 71 (1993) 3409-3412 hep-th/9308036.

[8] U. H. Danielsson, The deformed matrix model at finite radius and a new duality symmetry, Phys. Lett. B325 (1994) 33-38 hep-th/9309157.

[9] U. H. Danielsson, Two-dimensional string theory, topological field theories and the deformed matrix model, Nucl. Phys. B425 (1994) 261-276 hep-th/9401135.

[10] U. H. Danielsson, The scattering of strings in a black hole background, Phys. Lett. B338 (1994) 158-164 hep-th/9405052.

[11] U. H. Danielsson, A matrix model black hole: act II, JHEP 0402 (2004) 067 hep-th/0312203. 
[12] S. Gukov, T. Takayanagi and N. Toumbas, Flux backgrounds in 2D string theory, JHEP 0403 (2004) 017 hep-th/0312208.

[13] V. Kazakov, I. K. Kostov and D. Kutasov, A matrix model for the two dimensional black hole, Nucl. Phys. B622 (2002) 141-188 hep-th/0101011.

[14] J. Davis, L. A. Pando Zayas and D. Vaman, On black hole thermodynamics of 2-D type 0A, JHEP 0403 (2004) 007 hep-th/0402152.

[15] M. D. McGuigan, C. R. Nappi and S. A. Yost, Charged black holes in twodimensional string theory, Nucl. Phys. B375 (1992) 421-452 [hep-th/9111038|.

[16] N. Berkovits, S. Gukov and B. C. Vallilo, Superstrings in $2 D$ backgrounds with $R$-R flux and new extremal black holes, Nucl. Phys. B614 (2001) 195-232 hep-th/0107140.

[17] G. Gibbons, S. W. Hawking, Action integrals and partition functions in quantum gravity, Phys. Rev. D15 (1977) 2752-2756.

[18] H. Liebl, D. V. Vassilevich and S. Alexandrov, Hawking radiation and masses in generalized dilaton theories, Class. Quant. Grav. 14 (1997) 889-904 gr-qc/9605044.

[19] G. Gibbons, R. Kallosh, Topology, entropy and Witten index of dilaton black holes, Phys. Rev. D51 (1995) 2839-2862 hep-th/9407118.

[20] S. W. Hawking, G. T. Horowitz and S. F. Ross, Entropy, area, and black hole pairs, Phys. Rev. D51 (1995) 4302-4314 gr-qc/9409013.

[21] E. Witten, On string theory and black holes, Phys. Rev. D44 (1991) 314-324. 